\title{
A Constraint Manager \\ to Support Virtual Maintainability
}

\author{
Luis Marcelino \\ Norman Murray \\ Terrence Fernando \\ Centre for Virtual Environments, University of Salford \\ Salford, U.K. \\ \{L.Marcelino, T.Fernando, N.Murray@Salford.ac.uk
}

\begin{abstract}
Virtual prototyping tools have already captivated the industry's interest as a viable design tool. One of the key challenges for the research community is to extend the capabilities of Virtual Reality technology beyond its current scope of ergonomics and design reviews. The research presented in this paper is part of a larger research programme that aims to perform maintainability assessment on virtual prototypes. This paper discusses the design and implementation of a geometric constraint manager that has been designed to support physical realism and interactive assembly and disassembly tasks within virtual environments. The key techniques employed by the constraint manager are direct interaction, automatic constraint recognition, constraint satisfaction and constrained motion. Various optimisation techniques have been implemented to achieve real-time interaction with large industrial models.
\end{abstract}

\section{Keywords}

\section{INTRODUCTION}

Modern markets not only urge companies to create better products, but also force them to do so in increasingly shorter time scales. The concept of Concurrent Engineering (CE) encourages companies to address issues such as maintenance very early in the design process. However, the lack of simulation tools hinders the adaptation of $\mathrm{CE}$ and therefore further research is required to develop such tools. A development stage that is still lacking the appropriate software tools is maintenance simulation. The simulation of maintenance operations allows maintenance to be addressed in early design stages. This reduces unforeseen problems creeping into the design as it progresses through its life cycle, consequently saving both time and money while improving product quality.

The Virtual Prototyping Group at the Centre for Virtual Environments at Salford has been investigating the applicability of VR to interactive maintenance simulation. A system that can simulate realistic maintenance operations interactively is demanded by the industry. This research inverstigates the use of virtual environments to assess maintenance operations before any physical prototype is available. Besides speeding up the development process, the assessment of virtual models can also reduce the number of required physical prototypes. Such a tool has the potential to reduce the time-to-market and the development cost.
Maintenance operations are usually performed in a restricted space within a limited timespan. The operator's movements are often constrained by the surrounding components and contacts and clashes between components are inevitable. Furthermore, the time required for maintenance needs to be controlled and minimized. For these issues to be considered in a maintenance simulation, the computer simulation needs to be realistic.

To achieve a real-time realistic simulation we use a geometric constraint approach. Geometric constraints are relationships established between different geometric primitives. These relationships constrain the movement of objects and determine the objects' kinematics. The use of geometric constraints has two advantages against the alternative physical simulation: it is less computationally intensive, and only requires the information already available in CAD models. The more accurate physical simulation needs extra information like mass, friction coefficient, etc.

This paper describes the development and implementation of a Geometric Constraint Manager (CM). This constraint manager is being used in real-time immersive virtual environments such as the CAVE [Cruz-Neira-93] and the Workbench [Cutler97] to assess maintainability of virtual prototypes. The $\mathrm{CM}$ can recognize new constraints, enforce existing constraints and validate applied constraints. It is part of a more complex VR system [Fernando00] [Fernando01] [Murray02] that supports assem- 
assembly and disassembly operations in immersive virtual environments.

The next section introduces some of the work done in virtual maintainability. Section 3 describes some of this system's functionality and Section 4 presents its architecture and implementation. The simulation of maintenance tasks using a real industrial model required the constraint manager to be optimised as described in Section 5. The CM performance using the industrial case study is presented in Section 6. Section 7 discusses the achieved results and defines the orientation for future work.

\section{RELATED WORK}

There have been several research efforts to develop assembly simulator environments and much work has been done allowing users to compose scenes within virtual environments, such as 3DM [Butterworth-92], and JDCAD [Liang-94] which tackled many of the issues involved in the interactive creation of 3D objects. Problems with such systems are the absence of constraints when interacting with virtual objects. Users are restricted to gross interactions and are unable to perform precise object manipulation [Mine97]. Systems that support constraint based assembly of components provide the user with the support required to position components precisely in 3D space. There have been several research efforts to investigate the development of assembly simulation environments. For example, Connachar et al. [Connacher96] [Jayaram99] describes a system called VADE (Virtual Assembly Design Environment) interfaced with the Pro/Engineer modelling environment. A CAD database is connected to the Open Inventor API through Pro/Engineer Pro Develop Toolkit. In this system, direct interaction is supported through a CyberGlove. Fernando et al. [Fernando95], Fa et al. [Fa93], Munlin [Munlin95], and Thomson et al. [Thompson98], have developed an Interactive Constraint Based Assembly Modelling (ICBAM) environment to bring physical realism to the assembly simulation arena. Zachmann [Zach01] developed an assembly simulator that implement some constrained motion based on the type of contact between objects.

Fernando et al. [Fernando95] describes the methods developed for allowable motion and constraint recognition within the system. Automatic recognition of constraints such as 'against', 'coincident', 'tangential', and 'concentric' are supported in their system. By reading constraint relationships stored in a Relationship Graph, degrees of freedom can be computed so that the system can determine the allowable motions for a given assembly part. The VR environment is implemented using the IRIS Inventor graphical toolkit. The system described by Fernando et al. [Fernando95] reported some shortcomings. They include, inability to support constraint propagation from child object to parent when the child object is being manipulated in the assembly, and the absence of a stan- dard data translator for CAD data import into the virtual environment.

\section{SYSTEM DEFINITION}

This section presents the functionality of the constraint manager that was derived from its requirements. These requirements are based on previous experience building an assembly simulator [Wimalaratne01].

The development of a new constraint manager aimed to create an efficient and independent software toolkit that could be easily integrated into different virtual reality systems. The requirements for this system were:

- Multi-platform (UNIX and windows)

- Scene graph independence

- Multiple constraint recognition

- Multiple constraint satisfaction

- Deletion of broken constraints

- Automatic constraint management

The communication between the constraint manager and the main application uses a defined API with appropriate data structures. The internal data representation is based on private classes that are independent of any scene graph. For this reason the VR system needs to insert into the constraint manager each component's geometry, because the constraint manager has its own internal data representation. This allows the main application to choose the objects that can be constrained. The virtual hand, for example, would not be inserted into the constraint manager and therefore not be subject to geometric constraints.

The constraint manager has two types of geometry nodes: objects and surfaces. An object is an entity that has surfaces and can be moved. A surface is a face of an object. The constraint manager supports parametric surfaces and uses specific elements that define its parametric equation. For example a point on the plane and its normal vector defines a planar surface while a point on the cylinder axis, its direction and the radius of the cylinder value define a cylindrical surface. Besides surface specific elements, each surface has a bounding volume. This volume defines surface's borders because the equations define limitless surfaces. The constraint manager has no polygonal representation of surfaces because such a representation is not relevant to the constraint management process.

The constraint manager can recognize and apply different types of geometric constraints. A geometric constraint is rule that determines the relative motion between two surfaces. This rule reduces the degrees of freedom of the objects that contain the involved surfaces, constraining their motion. The constraint manager sup- 
ports the three different types of constraints illustrated in Figure 1.

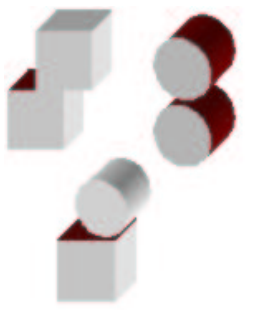

(a) Against

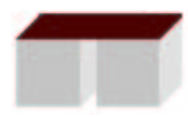

(b) Collinear

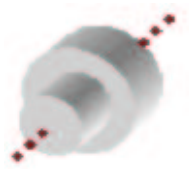

(c) Concentric

\section{Figure 1: Supported constraints}

The constraint recognizer must also be able to validate recognized and applied constraints. The validation is the process that determines whether a constraint is still valid or is broken. A constraint is broken if the involved surfaces attempt to move apart beyond a defined threshold.

The preferred way for the VR system to exchange data with the Constraint Manager is through the use of lists. The VR system can send to the constraint manager a list of surfaces with constraints to be added and the constraint manager can return a list of surfaces with the recognized constraints. Lists are a convenient communication medium because they do not restrict the amount of data passed to and from the constraint manager and a list received from the constraint manager can be freely manipulated by the application and then sent back to the constraint manager.

The functionality of the constraint manager can be divided in three main tasks:

- To validate existing constraints and determine the broken constraints;

- To enforce existing constraints and solve constrained motion;

- To recognize new possible constraints.

This functionality can be combined to achieve three stage fully automated constraint manager. Once a component's transformation is passed to the constraint manager, the model is searched for possible broken constraints that are removed. The remaining constraints are enforced and a resulting transformation computed. Once in a position, the constraints manager searched for new constraints between the moved component and the surrounding.

\section{SYSTEM ARCHITECTURE}

This section describes the architecture of the implemented constraint manager. Figure 2 shows a graphical representation of this architecture.

The constraint manager has a hierarchical data graph that maintains all relevant information from objects and surfaces. The data graph is available to all modules of the constraint manager: the constraint solver, the constraint recogniser and the filters. The constraint manager modules are independent and their interaction is defined by the application.

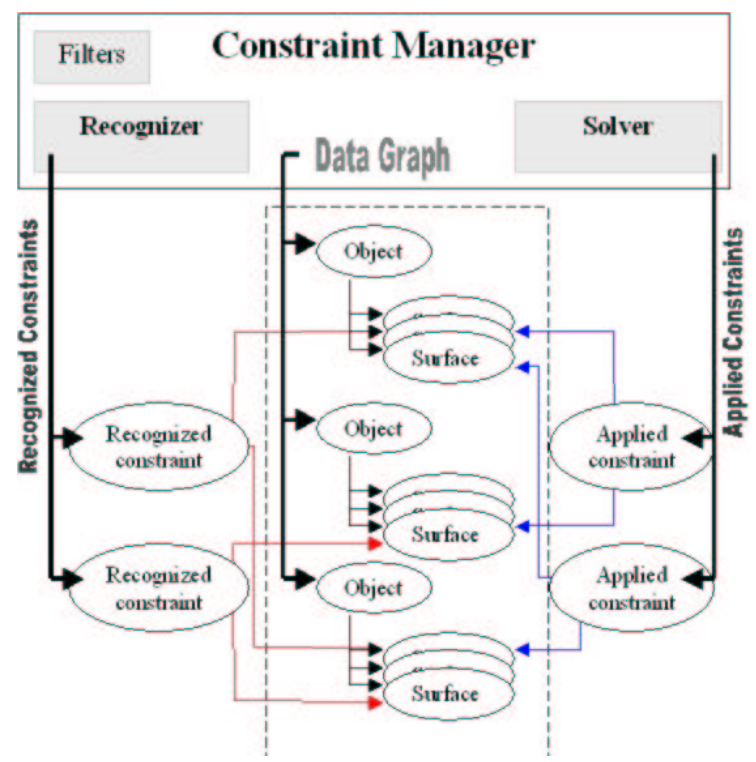

Figure 2: The Constraint Manager Architecture

\subsection{The data-graph}

The data graph is a hierarchical data structure that maintains the information relevant to the constraint manager. It represents all the knowledge the constraint manager has about the components within the virtual environment. This means the constraint engine has its own insight of the virtual environment. Virtual objects and surfaces that are not to be constrained are simply not added to the constraint engine and so are not considered during the solving and recognition process.

The data graph is organized like a flat scene-graph with the top-level nodes representing objects and the leafnodes representing surfaces. All objects and surfaces added to the constraint manager are in the data graph. A data graph's object is a node that has a list of surfaces and can be transformed. Valid transformations are translation and rotation, but not scale. An object can be transformed through direct manipulation or as a result of other object's movement. An object can be fixed in the 3D space to prevent it from being moved.

\subsection{The constraint solver}

The constraint solver is the module that manages applied geometric constraints. The solver determines the transformations to be applied to unfixed objects so that applied constraints are enforced. It is also the solver that applies new constraints, removes existing ones and fix objects in the $3 \mathrm{D}$ environment. Underlying this module is D-Cubed 3D DCM[dcubed01] library that computes the motion of constrained objects. 
The constraint solver maintains a list of the applied constraints. Applied constraints are the constraints to be enforced and that condition objects' transformations. Constraints apply to surfaces of distinct objects only, because this library only supports rigid bodies. This means that different surfaces of the same object are always fixed relative to each other and no constraint can be applied between them.

All transformations pass by the constraint solver. A new transformation for an object means that this object is being moved from its current position to a new one. The solver determines whether this motion is possible or not and, if not, it computes an alternative position. An object's motion can also affect the position of other constrained objects. The solver also computes the new position of constrained objects and updates these objects position.

\subsection{The constraint recognizer}

The constraint recognizer identifies new possible constraints and validates existing ones. The application specifies a list of objects to be searched for new constraints and possibly the surfaces to be tested for new constraints. If the application can determine collisions between surfaces, it can send those colliding surfaces to the constraint recognizer. This speeds up the recognition process because it cuts the number of surfaces to be tested.

The constraint recognizer has two lists of recognized constraints. Figure 2 shows only one of these lists for clarity. One list has the new recognized surfaces while the other has the existing constraints that failed to be recognized. These list of constraints are returned to the application, which then decides what to do with them. It is under the application's control to apply all, some or none of the recognized constraints, as it can control which constraints to break: all of those recognized, some of them or none of them.

The methods used for recognition of new constraints are also used to validate existing constraints. The validation process is based on the principle that a recognizable constraint is still a valid one. Validation takes place before existing constraints are enforced, otherwise existing constraints would always be recognized. Existing constraints that fail to be recognized are added to the list of broken constraint.

The constraint manager has a set of variables that define the tolerance of the recognition process. These tolerances determine the threshold under which constraints are recognized and can be adjusted dynamically by the application. The three recognition tolerances are the linear tolerance, the angular tolerance and the breaking factor. The linear tolerance is the maximum distance between two surfaces (or axis if two cylinders are involved), the angular tolerance is the maximum angle between two surfaces and the breaking factor is the scaling factor that multiplies the linear and angular tolerances when con- straints are being validated. A breaking factor greater than 1 means it is easier to recognize new constraints than to break existing ones. Increasing the breaking factor makes constraints more difficult to break.

The constraint recognition algorithm compares surfaces and verifies if their relative positions and orientations are within the specified tolerances. The application defines a list of surfaces that the recognizer uses to search possible constraints. Alternatively objects can be inserted into this list, in which case the recognizer searches all surfaces of those objects for possible constraints. The recognition process starts with a bounding box intersection test. To include the tolerance in the bounding box test, both surface boxes are enlarged by half the tolerance values. If bounding boxes are overlapping, the surfaces relative position and orientation is assessed to recognise possible constraints.

A constraint is recognized between two planes when the angle between their normals is less than the angular tolerance and that the distance between the planes is less than the linear tolerance. Two cylinders have a potential constraint when their axes make an angle within the angular tolerance and are less than the tolerance apart. The constraint between a plane and a cylinder is recognized if a plane's normal it perpendicular within the tolerance to the cylinder's axis and if the distance between the cylindrical and the planar surface is less than the linear tolerance.

\subsection{Filters}

Filtering is required to reduce the number of recognized constraints to a minimum. It takes three constraints to completely fix an object to other. Filters are functions that selectively remove recognized constraints from their list. The need for a filtering mechanism was raised when we tested the constraint manager with industrial case studies. These models do not have optimised surfaces and what could be one surface is sometimes a collection of small surfaces of the same type. As a result, recognition of geometric constraints often generates a large number of possible but redundant constraints. Filters reduce the constraint list according to their criteria and are mutually independent. The application chooses what filters to apply to the list of recognized constraints and in which order.

Some of the filters currently provided by the constraint manager include:

- Surface displacement: filters the constraint of a specified type that have closer surfaces;

- Cylinder radius: remove concentric constraints detected between cylinders of different radii;

- Cylinder definition: removes duplicated cylindrical constraints involving different cylindrical surfaces with identical geometry;

- Constraint type: remove all constraints of a specified type; 


\section{SYSTEM OPTIMISATION}

The use of the constraint manager with real industrial case studies revealed that the constraint manager did not scale up to support real industrial models. The models used by the industry are significantly more complex than the models used during the implementation of the constraint manager. The test model shown in Figure 3 involved assembling components with approximately 20 surfaces each while the industrial case study involved one

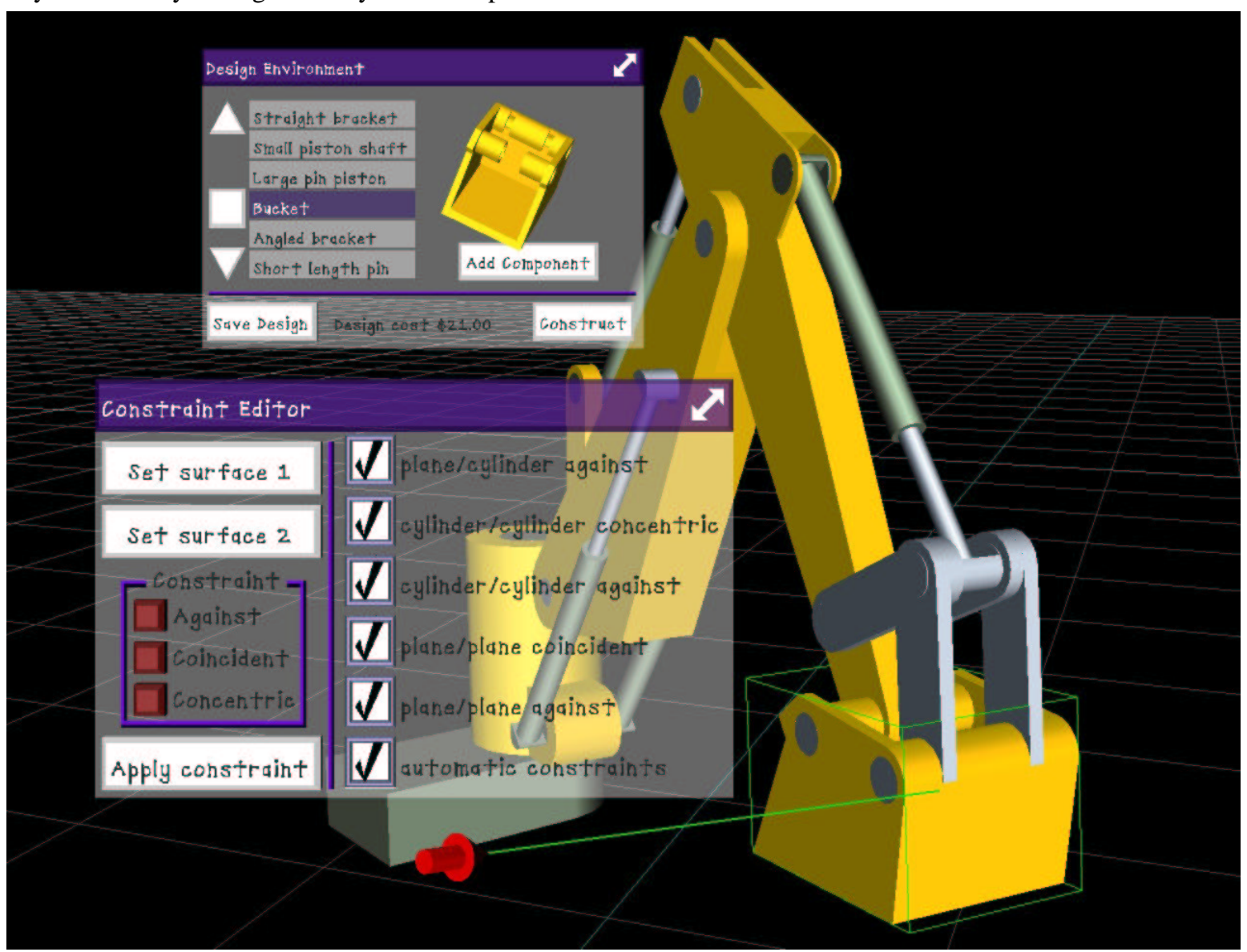

Figure 3: An interface to the Constraint Manager with the original model

component with more than 800 surfaces and others with more than 50 .

An assessment using the industrial case study revealed two bottlenecks in the constraint manager. One was the recognition process and the other was the transformation of objects. Using the industrial model the constraint manager needed nearly 200 milliseconds to recognize constraints and 80 milliseconds to move constrained objects. Surprisingly the constraint solver also needed approximately 70 milliseconds to move unconstrained objects. These two bottlenecks resulted in unacceptably low frame rate.

Several techniques were implemented to improve the performance of the constraint manager. This paper only presents the adopted solutions that are now part of the constraint manager.

\subsection{The recognizer optimisation}

The constraint recognizer was integrated in a VR framework that was adding pairs of objects, instead of pairs of surfaces, into the list to be recognized. The recognizer ognizer then created a list of surface pairs from all possible surfaces combinations. One object with $\mathrm{X}$ surfaces and another with $\mathrm{Y}$ surfaces resulted in $\mathrm{X}^{*} \mathrm{Y}$ surface pairs. Recognizing constraints between two components from the original model required 400 surface pairs to be tested while with the industrial case study this number increased to 40000 surface pairs.

To reduce the number of surface pairs to be tested, spatial information was added to each surface. This spatial information aimed at reducing the number of surfaces to be considered at a very low computational cost. For this reason we implemented a flat data structure of axis aligned cells. The advantage of axis aligned regular grids is their low computational load compared to oriented bounding boxes. This advantage is at expense of accuracy, but once the intention is to swiftly discard surfaces that are clearly beyond the region of interest, it offers a good compromise. Different bounding volumes could have been used which are described in the collision detection literature [Jiménez01]. 
The spatial filtering is a pre-processing step that is done when a component is added to the constraint manager. A bounding box is created for each component by adding the bounding boxes of all its surfaces. The component's bounding box is then divided into eight equal spatial cells and each surface is assigned to the spatial cells it uses.

Prior to recognising constraints between surfaces of two components the recognizer determines which cells of each component are intersecting. This information is then used to filter the surfaces pairs to be tested: only surfaces that are in intersecting cells are searched for possible constraints.

The association of spatial information to surfaces reduced considerably the number of surfaces to be considered in the recognition of new constraints. Using this new implementation the recognizer does in less than 30 milliseconds what previously took nearly 200 milliseconds for the chosen industrial case study.

\subsection{The solver optimisation}

All components are transformed using the constraint manager. The constraint manager receives a requested transformation and passes it to the solver. The solver determines the final transformation of objects enforcing applied constraints. The performance assessment showed that the 3D DCM library was using most of the time, even for unconstrained objects. The experiments also revealed that the time required to transform components depended more on the complexity of the components than on the applied constraints.

From the obtained results it was clear that to improve the solver performance we needed to reduce the number of surfaces inserted into the 3D DCM. All components added to the constraint manager are inserted into the DCM as bodies without surfaces. The data graph still maintains both objects and surfaces data and only information inserted into the 3D DCM library is simplified. Surfaces are only inserted into the DCM as required, i.e. when they are constrained. This way the DCM library is abstracted from the complexity of components and only deals with very simple bodies. As a result the performance of the constraint solver now depends on the number of applied constraints.

\section{EXPERIMENTAL RESULTS}

This section presents the performance results of the constraints manager using a real industrial case study. The three main processing stages are assessed.

The computer used to this experiments was an SGI ONYX2 with $128 \mathrm{MB}$ of RAM and two MIPS R10000 CPUs clocked at $180 \mathrm{Mhz}$. However, the constraint manager is single threaded and only one CPU was used at a time.

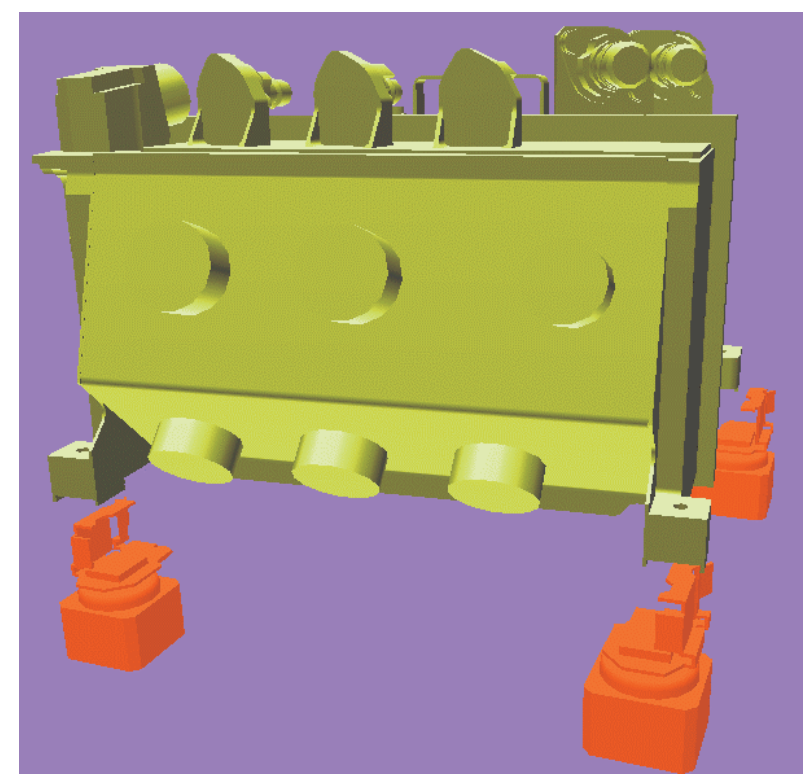

Figure 4: The Sener electronic box and its brackets

The model used in this experiment is a real case study from Sener, one of our industrial partners. The model has one electronic box and four brackets where it clamps. Some pipes and tubes are also part of the model but were not constrained. The electronic box is a component that has 872 surfaces and each bracket has 275 surfaces. This experiment consisted of the manipulation of the electronic box so it automatically recognizes constraints with one of the brackets. Once the electronic box clamps into the bracket it then moves along with the electronic box because it is not fixed within the world.

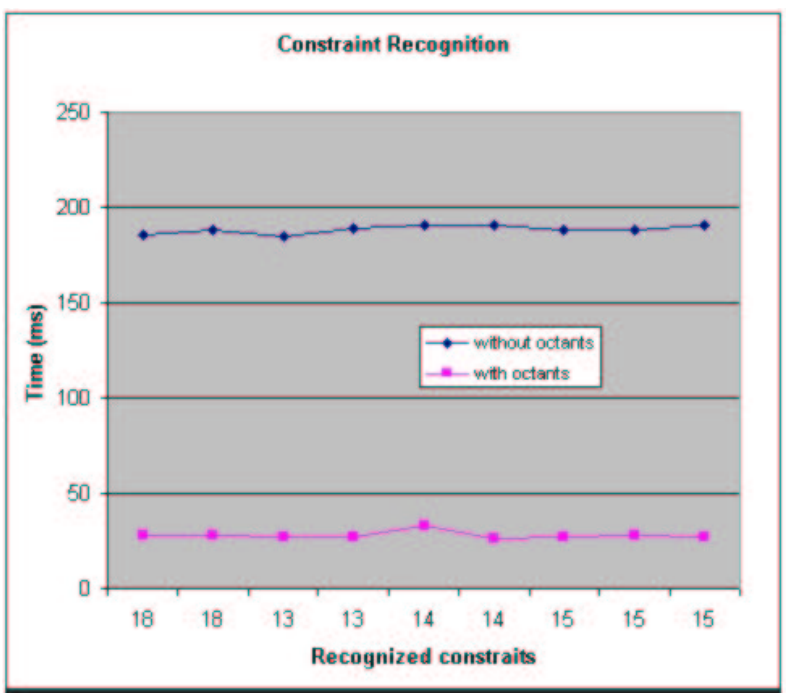

Figure 5: Automatic constraints recognition of two components with 872 and 275 surfaces.

The recognition process benefited significantly from adding spatial information to surfaces. Figure 5 shows that the time required to recognize constraints between the electronic box and a bracket was reduced from more than 180 milliseconds to 30 milliseconds. 


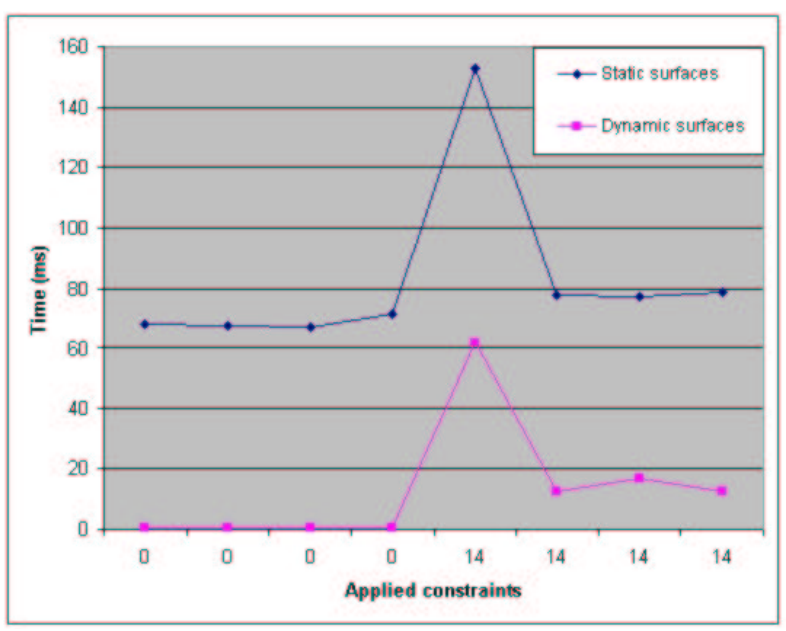

Figure 6: Time required transforming a component.

The time required to move objects was also reduced significantly due to the solver optimisation discussed in section 5.2. Figure 6 shows how the time to move the electronic box relates with the applied constraints. The "static surface" shows the time when all surfaces are inserted into 3D DCM while the "dynamic surface" when only constrained surfaces are inserted into the 3D DCM. Besides showing the performance improvement achieved by dynamically adding surfaces Figure 6 also shows the time 3D DCM needs when constraints are applied. This jump is due to the resetting of 3D DCM and happens every time constraints or geometries are added into, or removed from the library.

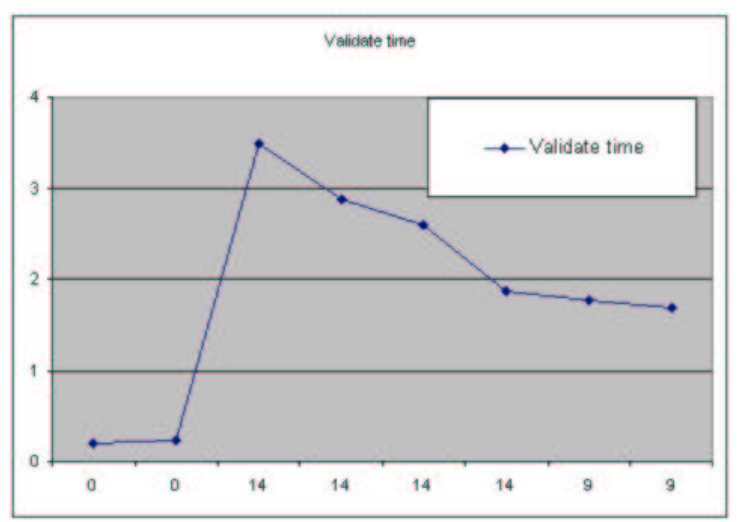

Figure 7: Time to validate existing constraints.

The validation time was inferior to the other two processing stages. Figure 7 shows that less than four milliseconds are needed to positively validate 14 existing constraints.

\section{CONCLUSIONS AND FUTURE WORK}

The results presented in the previous section demonstrate that the constraint manager can be used in interactive maintenance simulation of industrial models. The initial implementation of the constraint manager required approximately 250 milliseconds per interaction for fully automated constraint management and as a result could not be used interactively. The optimised ver- sion of the constraint manager needs approximately 50 milliseconds to do the same job. We find this is an acceptable simulation time given the complexity of the used components.

Despite the good results there is plenty of work to be done to achieve a mature system that can be used as a virtual prototyping tool. Further improvements to the existing constraint manager are now being considered. These improvements consist of developing a more efficient spatial data structure and applying filters before the recognition of new constraints.

\section{ACKNOWLEDGEMENTS}

This work has been supported by the EPSRC through a joint project with Rolls-Royce, EDS Parasolid and DCubed.

We would like to thank to Sener for providing the case study used in these experiments.

\section{REFERENCES}

[Butterworth-92] Butterworth, J., Davidson, A., Hench, S., Olano, T., "3DM: A Three-Dimensional Modeler Using a Head-Mounted Display", ACM Computer Graphics (1992 Symposium on Interactive 3D Graphics), Volume 25 (2), 1992, pp. 135-138

[Connacher96] Connacher, H. and Jayaram, S. and Lynos, K., "Integration of Virtual Assembly with CAD”, Symposium on Virtual Reality in Manufacturing Research and Education, October 1996, pp. 32-40

[Cruz-Neira-93] Cruz-Neira, C., Sandin, D., DeFanti, T., "Surround-Screen Projection-Based Virtual Reality: The Design and Implementation of the CAVE", Proceedings of the 20th annual conference on Computer graphics, Anaheim, USA, 1993, pp. 135-142

[Cutler97] Cutler, L and Froehlich, B. and Hanrahan, P., "Two-Handed Direct Manipulation on the Responsive Workbench", Proceedings of the 1997 Symposium on Interactive 3D Graphics, Providence, USA, April 1997

[dcubed01] D-Cubed, Ltd, "The 3D DCM Manual”, DCubed, Ltd, Version 2.5.0, August 2001

[Fa93] Fa, M., "Interactive Constraint-Based Assembly Modelling”, Ph.D. Thesis, School of Computer Studies, University of Leeds, Sept, 1993

[Fernando00] Fernando, T, Marcelino, L, Wimalaratne, P, Tan, K, "Interactive Assembly Modelling within a CAVE Environment", 9 Eurographics Portuguese Chapter Meeting, February 2000, Marinha Grande, pp.43-49

[Fernando01] Fernando, T., Marcelino, L., Wimalaratne, P., "Constraint-based Immersive Virtual Environment for Supporting Assembly and Maintenance Task", Proceedings of Human Computer Interaction International 2001, New Orleans, USA, August 2001 
[Fernando95], Fernando, T., Fa, M., Dew, P., Munlin, M., "Constraint-based 3D Manipulation Techniques for Virtual Environments", Virtual Reality Application, Chapter 6, Academic Press, 1995, pp. 71-89

[Jayaram99] Jayaram, S., Jayaram, U., Wang, Y., Tirumali, H., "Data Sharing and Control in AEC Software Integration", IEEE Computer Graphics and Applications, November/December 1999

[Jiménez01] Jiménez, P., Thomas, F., Torras, C., “3D Collision Detection: A Survey", Computers \& Graphics, 25(2), 2001, pp. 269-285

[Liang-94] Liang, J., Green, "JDCAD: A Highly Interactive 3D Modeling System", M. Computer \& Graphics, Volume 18 (4), 1994, pp. 499-506

[Mine97] Mine, M., “A Meta-CAD System for Virtual Environments", Computer-Aided Design, Volume 29 (8), 1997, pp. 547-553

[Munlin95] Munlin, M., "Interactive Assembly Modelling within a Virtual Environment", Ph.D. Thesis,
School of Computer Studies, University of Leeds, September, 1995

[Murray02] Murray, N., Fernando, T., Aouad, G., “A Virtual Environment for the Design Simulated Construction of Prefabricated Buildings, to appear in the Computer-Aided Civil and Infrastructure Engineering Journal

[Thompson98] Thompson, M.R, Maxfield, J.H., Dew, P.M "Interactive Virtual Prototyping”, Eurographics '98, April 1998, pp. 107-119

[Wimalaratne01] Wimalaratne, P., Fernando, T., "Supporting Assembly and Disassembly Operations through Direct Manipulation within a Virtual Prototyping Environment",8Th ISPE International Conference On Concurrent Engineering: Research And Applications, July 2001

[Zach01] Zachmann, G. and Rettig, A., "Natural and Robust Interaction in Virtual Simulation", Proceedings of the 8th ISPE international conference on concurrent engineering, Anaheim, USA, 2001 